Aus der Abteilung Hals-Nasen-Ohrenheilkunde

(Prof. Dr. med. Ch. Matthias)

im Zentrum Augenheilkunde und Hals-Nasen-Ohrenheilkunde

der Medizinischen Fakultät der Universität Göttingen

\title{
Struktur und Funktion der afferenten Synapse \\ innerer Haarzellen der Cochlea
}

\author{
INAUGURAL-DISSERTATION \\ zur Erlangung des Doktorgrades \\ der Medizinischen Fakultät
}

der Georg-August-Universität zu Göttingen

vorgelegt von

Alexander Meyer

aus Osnabrück

Göttingen 2010 
Dekan: Prof. Dr. med. C. Frömmel

I. Berichterstatter: Prof. Dr. med. T. Moser

II. Berichterstatter/-in:

III. Berichterstatter/-in:

Tag der mündlichen Prüfung: 


\section{Inhalt}

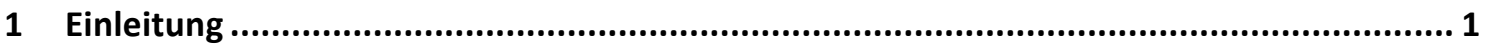

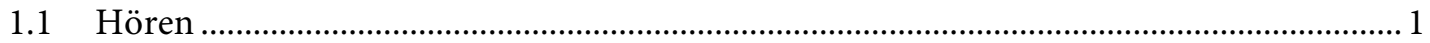

1.2 Innere Haarzellen und ihre afferente Synapse ……….................................................... 2

1.3 Calciumeinstrom und Transmitterfreisetzung …….......................................................... 3

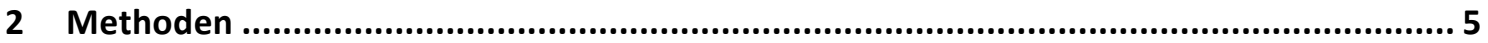

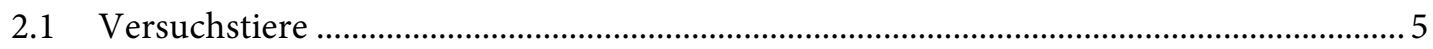

2.2 Elektrophysiologie ............................................................................................. 5

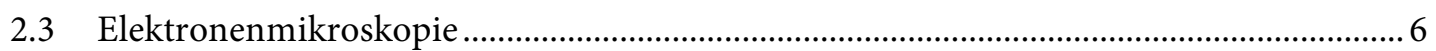

2.4 Konventionelle optische Mikroskopie …………............................................................. 6

2.5 Höchstauflösende optische Mikroskopie........................................................................... 7

2.6 Funktionelles Calcium-Imaging.................................................................................. 8

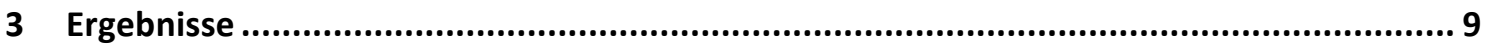

3.1 Tonotope Karte der Innervationsdichte innerer Haarzellen ..........................................

3.2 Verteilung der Synapsen in der Haarzelle ........................................................................ 10

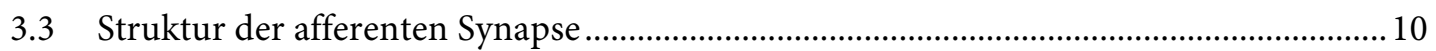

3.4 Höchstauflösende optische Mikroskopie ......................................................................... 10

3.5 Funktion der afferenten Synapse ............................................................................ 11

3.6 Calcium-binding Proteins und calciumabhängige Calciumstrominaktivierung ............. 12

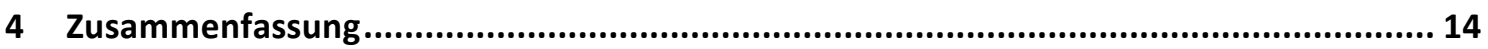

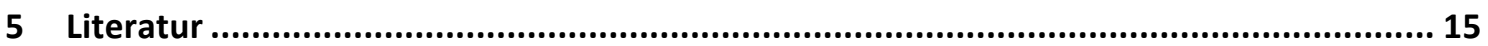

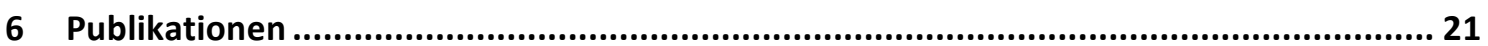

Publikation I: Meyer et al., 2009.

Publikation II: Cui et al., 2007. 


\section{$1 \quad$ Einleitung}

\section{$1.1 \quad$ Hören}

Die Fähigkeit, Schwingungen des sie umgebenden Mediums wahrzunehmen, haben Lebewesen bereits frühzeitig in der Entwicklungsgeschichte erworben. Diese mechanischen Schwingungen - wir sprechen in einem definierten Frequenzbereich von Schall - tragen Informationen über die unmittelbare, aber auch weitere Umgebung. Ihre Wahrnehmung ist für fast alle höheren Lebewesen unverzichtbar, um erfolgreich auf ihre Umwelt reagieren zu können. Form und Funktion der Organe, die sich im Laufe der Evolution zur Schallwahrnehmung entwickelt haben, sind in ihrer Konstruktion so vielfältig wie die Spezies, die sie tragen, selbst. Sie besitzen jedoch gemeinsame Bauprinzipien, die sich exemplarisch am Ohr der Säugetiere wiederfinden. Dessen Wahrnehmungsleistung kann in jeder Hinsicht als außergewöhnlich gelten. Der Umfang der hörbaren Frequenzen beispielsweise beim Menschen beträgt drei Größenordnungen, der der Intensitätswahrnehmung sogar bis zu sieben. Die innerhalb des aufgespannten Hörfelds erreichte Zeitauflösung erreicht den Sub-Millisekundenbereich, Frequenz- und Intensitätsauflösung stehen dieser Leistung kaum nach. Erst diese Fähigkeiten des Gehörs ermöglichen so komplexe Prozesse wie beispielsweise Richtungslokalisation oder Sprachverstehen.

Was befähigt dieses Organ zu einer solchen Leistung? Das Ohr ist eines der strukturell kompliziertesten Organe, die wir kennen. Mit dem der Umgebung zugewandten äußeren Ohr, welches den Schall aufnimmt, filtert und bündelt, dem Mittelohr als Wandler von Luft- in Flüssigkeitsschwingungen, dem Innenohr als Verstärker und Umsetzer in neuronale Signale und schließlich dem Hörnerv, der Hörbahn und dem auditorischen Cortex, die die Nervenimpulse leiten, verarbeiten und interpretieren, verfügt das auditorische System über eine Signalkette, deren Glieder darauf ausgelegt sind, auf jeder Stufe einen Teil der Verarbeitung zu übernehmen und gleichzeitig die relevanten Details des Eingangssignals zu erhalten.

Die mit dieser Arbeit vorgelegten Publikationen beschäftigen sich mit der Funktion der Cochlea, genauer gesagt mit jenen funktionellen Einheiten, die als Teil des Corti'schen Organs die Umsetzung mechanischer Schwingungen in Nervenimpulse vermitteln: den inneren Haarzellen (IHZ). Es soll die Frage beleuchtet werden, in welcher Form deren Struktur und Funktion zur Signalverarbeitung und -weiterleitung beitragen.

Von Bekesy (1960) konnte zeigen, daß durch die mechanischen Eigenschaften der Cochlea eine Frequenzdispersion der Schallwellen entlang der Schnecke auftritt, die dazu führt, daß Schallsignale je nach ihrer Frequenz an definierten Stellen der Cochlea die Basilarmembran und damit die IHZ zu Schwingungen anregen. Äußere Haarzellen (ÄHZ) tragen zur Verstärkung und Schärfung der Frequenztrennung bei (Ryan und 
Dallos, 1975; Kiang et al., 1976; Dallos und Harris, 1978; Drexl et al., 2008; Cheatham et al., 2009).

Innere Haarzellen sind tonotop, d.h. von der Basis der Cochlea absteigend zum Apex nach Frequenz angeordnet. Die Tonotopie spiegelt den ersten wichtigen Schritt der Signalverarbeitung wider. Dies wird durch die Tatsache unterstrichen, daß das Tonotopieprinzip in fast allen Teilen der Hörbahn - peripheren (Liberman, 1982b; Müller, 1996; Müller et al., 2005) wie zentralen Anteilen (Møller, 2006) - verfolgt wird.

\subsection{Innere Haarzellen und ihre afferente Synapse}

Wie sieht die Umsetzung an den IHZ im Detail aus? Mechanosensitive (MET, MEchano Transduktions-) Kanäle an den namensgebenden feinen Härchen (Stereozilien) am apikalen Pol der IHZ führen bei Auslenkung durch Schallschwingungen zu einer graduierten Depolarisation der Zelle (Howard et al., 1988; Hudspeth, 1989; Fettiplace, 2009). Der basale Pol der IHZ beherbergt die Synapsen, die Kontakte zu den afferenten bipolaren Spiralganglienneuronen (SGN) des Hörnerven formen. Die Synapsen gehören zu den sogenannten Bändersynapsen (engl. ribbon synapses), die sich in ihrer Struktur von Synapsen des ZNS unterscheiden. Namensgebend ist eine präsynaptische elektronendichte Struktur, das synaptische Band oder Ribbon, das sich in variabler Größe und Form in das Innere der Zelle fortsetzt (siehe Moser et al., 2006, für eine Übersicht). Angeheftet daran finden sich synaptische Vesikel, die auf die Freisetzung ihres Inhalts in den synaptischen Spalt warten. Signal für die Freisetzung ist der Einstrom von Calciumionen durch $\mathrm{Ca}_{\mathrm{v}} 1.3-\mathrm{L}-\mathrm{Typ}$-Calciumkanäle (Platzer et al., 2000; Brandt et al., 2003; Brandt et al., 2005) und deren Bindung an den Freisetzungsapparat.

Bändersynapsen finden sich außer in cochleären auch in vestibulären Haarzellen und in Photorezeptorzellen und Bipolarzellen der Retina (Übersicht in Sterling und Matthews, 2005). Diesen Zellen ist gemein, daß sie als Sinneszellen graduelle Rezeptorpotentiale entwickeln und daher über einen langen Zeitraum eine hohe Rate der Transmitterausschüttung aufrecht erhalten müssen.

Bändersynapsen verwenden einheitlich Glutamat als Transmitter. Dieser diffundiert nach Freisetzung durch den synaptischen Spalt und bindet an Rezeptoren, die in den dendritischen Endigungen der postsynaptischen Spiralganglienneurone verankert sind. Diese bilden an den synaptischen Kontaktstellen zu den inneren Haarzellen Auftreibungen, sog. Boutons (Liberman, 1980, 1982a). IHZ werden von mehreren SGN innerviert, wobei jedes SGN jedoch lediglich eine einzige IHZ kontaktiert (Spoendlin, 1969; Liberman, 1980; Kiang et al., 1982; Spoendlin, 1985). In Boutons von Meerschweinchen, Maus und Ratte sind insbesondere Glutamatrezeptoruntereinheiten vom AMPA-Typ GluR2/3 und GluR4 nachgewiesen worden (Ruel et al., 2000; Eybalin et al., 2004; Khimich et al., 2005). 
Die Signalübertragungseigenschaften der afferenten SGN sind in vielen Säugetierspezies im Detail untersucht worden (Tasaki, 1954; Evans, 1972; Dallos und Harris, 1978; Liberman, 1978; Ohlemiller und Echteler, 1990; Taberner und Liberman, 2005). Bestimmte Eigenschaften der Übertragung zeigen sich dabei speziesübergreifend, so daß man glaubt, diese als grundlegende Prinzipien des peripheren auditorischen Systems verstanden zu haben:

Fundamentale Eigenschaft einer auditorischen Nervenfaser ist ihre Charakteristische Frequenz (CF), die dem Minimum der frequenzabhängigen Abstimmkurve (oder auch Schwellenkurve, engl. tuning curve) entspricht. Die in Hörnervenfasern von Säugetieren gemessenen tuning curves (Kiang et al., 1965; Taberner und Liberman, 2005) weisen typischerweise ein scharf begrenztes Minimum auf, was eine hohe Frequenzselektivität der einzelnen Faser bei geringen Lautstärken belegt.

Fasern gleicher CF zeigen in Abwesenheit von Stimulation erhebliche Unterschiede in ihrer Spontanaktivität. Das Spektrum kann dabei je nach Spezies multimodal verteilt sein (z.B. in der Katze) und damit in Klassen von hoher und niedriger Spontanaktivität fallen (Kiang et al., 1965; Liberman, 1978) oder - wie in der Maus - kontinuierlich verteilt sein (Taberner und Liberman, 2005). In direkter Korrelation zur Spontanaktivität steht die Schwelle an der CF, wobei solche mit hoher Spontanaktivität eine niedrige Aktivierungsschwelle besitzen und umgekehrt. Eine Faser mit niedriger Aktivierungsschwelle wird außerdem bei vergleichsweise niedrigen Schallpegeln ihre maximale Entladungsfrequenz erreichen und so einen anderen Schalldruckbereich abdecken als Fasern hoher Aktivierungsschwelle (Taberner und Liberman, 2005). Eine Gruppe verschiedener Fasertypen, die eine IHZ innerviert, kann so einen großen dynamischen Bereich abbilden.

Klassische, kombiniert elektrophysiologisch-morphologischen Studien am Corti'schen Organ der Katze berichteten über eine unterschiedliche Lokalisation der afferenten IHZ/SGN-Synapse je nach Faserklasse (Liberman, 1982a; Liberman et al., 1990; Merchan-Perez und Liberman, 1996). Fasern niedriger Spontanaktivität / Empfindlichkeit bilden demnach präferentiell Synapsen an der neuralen, modiolus-nahen Seite der IHZ, Fasern hoher Spontanaktivität / Empfindlichkeit eher auf der abneuralen, pfeilerzellnahen Seite. Die funktionelle Bedeutung dieses Befundes ist jedoch unklar, ebenso ist ungeklärt, ob eine ähnliche Ordnung auch in Spezies mit einer kontinuierlichen Faserverteilung besteht. Auf diese Frage wird in der ersten vorgestellten Publikation eingegangen.

\subsection{Calciumeinstrom und Transmitterfreisetzung}

Wie eingangs erwähnt, führt die mechanische Auslenkung der Stereozilien zu einem Einstrom von Kationen durch die MET-Kanäle an deren Spitze und Depolarisation der Membran (Übersicht in Fettiplace, 2009). Auslöser für die Freisetzung von Glutamat aus synaptischen Vesikeln ist der auf die Depolarisation der Zelle folgende Einstrom von Calcium durch präsynaptische Calciumkanäle. Im Gegensatz zu zentralen 
Neuronen, die im wesentlichen P/Q-, N- oder R-Typ Kanäle besitzen, finden sich in der präsynaptischen Membran innerer Haarzellen vor allem L-Typ-Kanäle vom $\mathrm{Ca}_{\mathrm{v}} 1.3$-Typ (Platzer et al., 2000; Brandt et al., 2003), die eine schnelle Kinetik mit ungewöhnlich geringer calciumabhängiger Calciumstrominaktivierung aufweisen und bei sehr negativen Potentialen aktivieren (Xu und Lipscombe, 2001).

Die Beziehung zwischen Calciumeinstrom bzw. intrazellulärer Calciumkonzentration und der Freisetzungsrate synaptischer Vesikel ist in IHZ (Beutner et al., 2001) wie auch zentralen Neuronen (Schneggenburger und Neher, 2000) hochgradig nichtlinear. Die Calciumbindung an den Freisetzungsapparat läßt sich mit Modellen beschreiben, die zwischen drei und fünf kooperative Bindungsstellen fordern. Das bedeutet gleichzeitig, daß eine geringe Änderung des Calciumeinstroms zu erheblichen Unterschieden in der Freisetzung führen kann. Eine Regulation des Calciumeinstroms ist somit ein effektiver Angriffspunkt für die Regulation der Transmitterfreisetzung. Es ist eine ganze Reihe von Mechanismen zur Modulation und Regulation von präsynaptischen Calciumkanälen bekannt. Dazu gehören beispielsweise Interaktion mit dem SNAREKomplex, G-Protein-vermittelte Modulation oder $\mathrm{Ca}^{2+} /$ Calmodulin-vermittelte Regulation (Catterall und Few, 2008). Die zweite der in dieser Arbeit vorgestellten Publikationen beschäftigt sich mit einem Mechanismus der Calciumstrom-Regulation, der am Effekt der calciumabhängigen Inaktivierung angreift.

$\mathrm{Ca}_{\mathrm{v}} 1.3$-Calciumkanäle weisen in heterologen Expressionssystemen eine ausgeprägte calciumabhängige Inaktivierung (engl. $\mathrm{Ca}^{2+}$-dependent inactivation, $\mathrm{CDI}$ ) auf (Koschak et al., 2001; Xu und Lipscombe, 2001; Song et al., 2003; Cui et al., 2007). Dieser Mechanismus wird durch Bindung von Calmodulin an die IQ-Domäne der Cav1$\alpha_{1}$-Untereinheit verursacht (Peterson et al., 1999; Qin et al., 1999). Auffälligerweise findet sich bei den $\mathrm{Ca}_{\mathrm{v}} 1.3$-gebundenen Calciumströmen der inneren Haarzellen deutlich weniger CDI als nach Messungen in heterologen Expressionssystemen zu erwarten gewesen wäre (Platzer et al., 2000; Michna et al., 2003; Schnee und Ricci, 2003; Song et al., 2003; Cui et al., 2007). Arbeiten der letzten Jahre lieferten Hinweise darauf, daß dieser Effekt durch eine Klasse von Proteinen vermittelt wird, die strukturelle Ähnlichkeit zu Calmodulin besitzen und dieses von seiner Bindungsstelle am Calciumkanal verdrängen können, ohne jedoch CDI zu vermitteln - die calcium-binding proteins (CaBP1-5, Haeseleer et al., 2000; Lee et al., 2002; Zhou et al., 2004; Yang et al., 2006). Unsere Arbeit behandelt die Frage, welche Mitglieder dieser Proteinfamilie in inneren Haarzellen vorkommen und die Hemmung der CDI vermitteln. 
Eine detaillierte Beschreibung der verwendeten Methoden findet sich in den jeweiligen Publikationen bzw. dem über die Homepage des Journals online verfügbaren Supplementary Material. Im Folgenden sollen die Methoden kurz vorgestellt und ihre Bedeutung für die vorgelegten Arbeiten erläutert werden.

\section{$2.1 \quad$ Versuchstiere}

Die Untersuchung von Cochleae von Nagetieren hat eine lange Tradition in der Hörforschung, was - abgesehen von deren relativ einfacher Haltung - an der prinzipiellen Ähnlichkeit zum menschlichen Hörorgan liegt. Wir haben zwei Spezies für unsere Untersuchungen ausgewählt, die wir für besonders geeignet halten, um an ihnen Mechanismen exemplarisch aufzuklären:

Die meisten Versuche wurden an Cochleae von Mäusen durchgeführt. In den letzten Jahren ist das Hörorgan der Maus in seiner grundlegenden Struktur und Funktion Gegenstand einer Reihe von Publikationen gewesen (Moser und Beutner, 2000; Müller et al., 2005; Taberner und Liberman, 2005, um nur einige wenige wichtige zu nennen). Wichtige Erkenntnisse sind nicht zuletzt durch Untersuchungen an mutanten Mäusen erlangt worden (Platzer et al., 2000; Roux et al., 2006; Cui et al., 2007; Neef et al., 2009); gerade die Möglichkeit gezielter genetischer Modifikation eröffnet der Forschung an dieser Spezies neue Wege (siehe Mullen und Ryan, 2001 für eine Übersicht).

Wir untersuchten Tiere der Linien Naval Medical Research Institute (NMRI) und C57/Black 6. Gerade von letzteren ist die frühzeitige Entwicklung einer Presbyakusis bekannt (Henry und Lepkowski, 1978; Willott, 1986). Aus diesem Grund wurden Tiere nur bis zu einem Alter von P30 verwendet.

Nehmen Mäuse Frequenzen vor allem im Hochtonbereich (1-100 kHz, Bereich des besten Hörens zwischen 12 und $32 \mathrm{kHz}$, Müller et al., 2005) wahr, so liegt das Hörfeld der mongolischen Wüstenrennmaus mit einem Bereich des besten Hörens zwischen 1 und $10 \mathrm{kHz}$ eher im Mittelfrequenzbereich (Müller, 1996) und somit nahe dem des Menschen. Wir haben daher Daten in beiden Spezies erhoben und verglichen.

\subsection{Elektrophysiologie}

Mit Einführung der Patch-clamp-Technik (Hamill et al., 1981; Sakmann und Neher, 1995) wurde es möglich, selbst kleinste Ionenströme über die Membran einer Zelle mit hohem Signal-zu-Rausch-Abstand und hoher zeitlicher Auflösung zu messen. Die weite Verbreitung dieser Technik beruht nicht zuletzt auch darauf, daß sich hiermit eine unüberschaubare Anzahl unterschiedlicher Zelltypen untersuchen läßt. Auch in- 
nere Haarzellen sind der Patch-clamp-Technik z.B. in whole-cell oder perforated patch Konfiguration zugänglich (Kros et al., 1998; Moser und Beutner, 2000). Es ist sogar möglich, die von Lindau und Neher (1988) beschriebene Methode der Abschätzung von Exo- und Endozytose über eine Messung der elektrischen Kapazität der Zellmembran zu verwenden (Moser und Beutner, 2000). Neuere Arbeiten, in denen erstmals auch Patch-clamp-Ableitungen von postsynaptischen Boutons erfolgreich durchgeführt wurden (Goutman und Glowatzki, 2007; Li et al., 2009), sprechen dafür, daß der Anstieg der Zellkapazität - zumindest kurz nach Beginn der Stimulation - gut mit der Menge an freigesetztem Transmitter korreliert.

Innere Haarzellen exprimieren eine Reihe verschiedener Ionenkanäle, allen voran spannungsabhängige L-Typ-Calciumkanäle vom Cav1.3-Subtyp (Brandt et al., 2003), calciumabhängige Kaliumkanäle (Kros et al., 1998) und die MET-Kanäle der Stereozilien (Howard et al., 1988; Fettiplace, 2009). Für die Messung des Calciumeinstroms mußte dieser durch pharmakologische Blockierung insbesondere der $\mathrm{K}^{+}$-Ströme isoliert werden.

\section{Elektronenmikroskopie}

Transmissions-elektronenmikroskopische Untersuchungen können Strukturen in der Größenordnung von Nanometern darstellen. Viele Teile der Nano-Maschinerie des präsynaptischen Freisetzungsapparates sind auf dieser Ebene zu erkennen, so beispielsweise der synaptische Ribbon oder transmittertragende Speichervesikel (Liberman et al., 1990; Khimich et al., 2005).

Wir führten in Zusammenarbeit mit D. Riedel, MPI für biophysikalische Chemie, Göttingen, eine elektronenmikroskopische Quantifizierung der Dimensionen präsynaptischer Elemente durch. Die für eine elektronenmikroskopische Darstellung notwendige chemische Fixation (zu den Details s. Meyer et al., 2009, Supplementary Material) ist jedoch mit dem Problem der Schrumpfung behaftet. Es wurden daher Vergleichsmessungen mit der alternativen Hochdruck-Gefrierfixation (high pressure quick freeze, HPF) durchgeführt, ohne jedoch relevante Unterschiede (Schrumpfung bei chem. Fixation $<10 \%$ ) festzustellen.

\subsection{Konventionelle optische Mikroskopie}

Auch wenn das Auflösungsvermögen der Elektronenmikroskopie nach wie vor unerreicht durch andere Techniken ist, gibt es zwei wesentliche Einschränkungen: Zum einen wirken Strukturen nach ihrer Elektronendichte kontrastgebend. Das führt dazu, daß vor allem größere Proteinkomplexe wie beispielsweise der Ribbon zur Darstellung kommen. Elemente, die möglicherweise für die Funktion der Synapse wichtig sind, jedoch nur in geringer Dichte vorkommen, werden nicht sichtbar. Zum anderen ist die Untersuchung an dünnen Schnitten extrem aufwendig, daher ist die Zahl der untersuchten Synapsen überschaubar - für eine aussagefähige Statistik kann dies ein Problem darstellen. 
Lichtmikroskopische Techniken haben diese Nachteile nicht. Mittels Immunfärbung lassen sich gezielt einzelne Elemente des synaptischen Komplexes fluoreszierend markieren. Wir haben so beispielsweise durch Wahl eines präsynaptischen und eines postsynaptischen Zielproteins eine verläßliche Darstellung und Zählung einer großen Zahl von Synapsen realisiert.

Problematisch ist jedoch, daß selbst in den modernsten konfokalen Mikroskopen die Auflösung durch die Beugung des Lichts begrenzt ist. Ernst Abbe (1873) formulierte das physikalische Gesetz zur Grenze des Auflösungsvermögens von Lichtmikroskopen. Danach kann ein Lichtmikroskop gerade noch Objekte voneinander trennen, deren Abstand der Wellenlänge des benutzten Lichtes entspricht, geteilt noch durch einen Faktor, der das Objektiv beschreibt, die sogenannte Numerische Apertur. Unter optimalen Bedingungen kann so eine Auflösung von etwa 200 Nanometern erreicht werden. Konfokale Mikroskope (Übersicht in Pawley, 2006) erreichen diese theoretische Auflösungsgrenze in der Tat durch ein Ausblenden der nicht zur Bildgebung beitragenden Strahlen außerhalb des Focus. Dies reicht zwar für eine qualitative Darstellung synaptischer Strukturen aus, für quantitative Messungen der Form und Ausdehnung einzelner Teile einer Synapse ist es jedoch zu grob.

\section{Höchstauflösende optische Mikroskopie}

Vor wenigen Jahren wurden nun von Prof. Stefan Hell vom Max-Planck-Institut für biophysikalische Chemie in Göttingen zwei Techniken entwickelt, die es erlauben, Fluoreszenzmikroskope mit Auflösungen weit jenseits der Abbeschen Grenze zu bauen (Hell und Wichmann, 1994; Hell, 2007; Hell et al., 2009).

Die sogenannte 4Pi-Mikroskopie beruht auf dem Prinzip, daß, anders als bei konventionellen konfokalen Mikroskopen, das Fluoreszenzlicht der zu untersuchenden Probe von zwei sich gegenüberliegenden Objektiven aufgefangen und kohärent registriert wird. Als Resultat ergibt sich ein Interferenzmuster entlang der Objektivachse (zAchse), aus dem sich mittels Dekonvolution ein Bild mit einer Auflösung von 70 $140 \mathrm{~nm}$ errechnen läßt. Damit steigt die Auflösung entlang der z-Achse von etwa $500 \mathrm{~nm}$ eines konventionellen konfokalen Mikroskops um den Faktor 4 - 7. Eine Einschränkung der 4Pi-Mikroskopie ist jedoch, daß sie die höchste Auflösung nur in einer Achse erbringen kann. Dies ist bei der zweiten Technik anders:

Die STED-Mikroskopie ermöglicht es, Fluoreszenzmikroskopie mit einer 2- oder 3 dimensionalen Auflösung theoretisch bis unterhalb von 50 - $100 \mathrm{~nm}$ zu betreiben. Dabei ist - wie bei der konfokalen Mikroskopie - lediglich ein Objektiv notwendig. Das Abbildungsverfahren beruht auf dem Prinzip der stimulated êmission depletion (der stimulierten Fluoreszenzabregung, STED). Danach ist es möglich ein Fluoreszenzfarbstoff-Molekül, das durch Licht angeregt wurde, durch Licht einer bestimmten Wellenlänge wieder abzuregen (depletion), ohne daß Fluoreszenzlicht abgestrahlt wird. Ein STED-Mikroskop verfügt daher über zwei Laser: Einen zur Anregung der Probe sowie einen zur Auslöschung der Anregung. Durch einen optischen Trick be- 
kommt dabei der Fokuspunkt des STED-Lasers die Form eines Rings, so daß vor allem Fluoreszenz in der Peripherie des Anregungsspots ausgelöscht wird. Im Zentrum des Rings verbleibt ein kleines fluoreszierendes Volumen, das zur Abbildung mit hoher Auflösung beiträgt. Dieses Verfahren ist auch auf drei Dimensionen erweiterbar, indem man dem Fokus des STED-Lasers die Form einer Kugelschale gibt. So ist eine Abbildung mit einer Auflösung von unter $150 \mathrm{~nm}$ in $\mathrm{x}$-, $\mathrm{y}$ - und $\mathrm{z}$-Richtung möglich.

\subsection{Funktionelles Calcium-Imaging}

Immunhistochemische Untersuchungen setzen voraus, daß das Gewebe fixiert wird und somit alle Abläufe in der Zelle zum Halten kommen. Funktionelle Untersuchungen sind daher nur an der lebenden Zelle möglich, aber auch hier können konfokale mikroskopische Techniken angewendet werden. Für die Freisetzung von Neurotransmitter ist der Einstrom von Calciumionen nach Depolarisation der Zellmembran das entscheidende Signal. Tucker und Fettiplace (1995) konnten mit Hilfe von Calciumindikatorfarbstoff, der über eine Patch-Pipette in das intrazelluläre Kompartiment eingebracht wurde, sowie eines konfokalen Scanners die präsynaptischen Mikrodomänen von Calcium nach Stimulation in Haarzellen der Schildkröte zur Abbildung bringen. Wir haben das Verfahren auf innere Haarzellen der Maus angewendet (Frank et al., 2009; Meyer et al., 2009). 


\section{Ergebnisse}

\section{Was unterscheidet innere Haarzellen an verschiedenen Stellen der Cochlea?}

Ausgangspunkt der ersten hier vorgelegten Publikation (Meyer et al., 2009) war der Befund, daß die cochleäre Funktion tonotope Unterschiede zeigt. Typischerweise findet sich in der Mitte des Hörfelds eine niedrige Hörschwelle, die zu den Rändern, d.h. den sehr tiefen und sehr hohen Frequenzen hin ansteigt (Moore und Hine, 1992; Müller, 1996; Uzuka et al., 1996; Zheng et al., 1999; Ngan und May, 2001; Wolski et al., 2003). Wie unterscheiden sich nun innere Haarzellen des empfindlichen, anatomisch in der Mitte der Cochlea gelegenen Bereichs in ihrer Struktur und Funktion von jenen an Apex und Basis?

\subsection{Tonotope Karte der Innervationsdichte innerer Haarzellen}

Wie eingangs erwähnt besitzen innere Haarzellen eine variable Anzahl an Synapsen und afferenten Nervenfasern (Liberman, 1980; Kiang et al., 1982; Spoendlin, 1985). Nicht sicher bekannt war bislang, ob ein Zusammenhang zwischen dem tonotopen Ort einer inneren Haarzelle und der Anzahl der sie versorgenden Spiralganglienneurone besteht (Francis et al., 2004; Stamataki et al., 2006). Um nun eine Karte der Innervationsdichte der Cochlea zu erstellen, markierten wir einzelne Bausteine der Synapsen immunhistochemisch. Wir wählten als Marker der präsynaptischen Seite das Protein RIBEYE (Schmitz et al., 2000), einer der Hauptbestandteile des synaptischen Ribbons, sowie die postsynaptischen Glutamatrezeptoruntereinheiten GluR2/3. Untersucht in einem konfokalen Mikroskop konnten Synapsen gegenüber unspezifischer Färbung als kolokalisierte Immunfluoreszenzpunkte identifiziert werden. Zusätzlich erfolgte noch eine Färbung der Zellkerne mit dem Farbstoff Hoechst 34580 zur Quantifizierung der Zahl der inneren Haarzellen.

Es wurden Färbungen in den Cochleae von Mäusen und mongolischen Wüstenrennmäusen durchgeführt. Wir waren damit in der Lage, eine Karte der Cochlea zu erstellen, der die durchschnittliche Zahl der Synapsen pro innerer Haarzelle in Abhängigkeit von ihrer Position in der Cochlea und somit ihrer charakteristischen Frequenz zu entnehmen ist. Faszinierenderweise folgt die Innervationsdichte dabei genau der Empfindlichkeitskurve der Schallwahrnehmung: In den unempfindlicheren Randbereichen des hörbaren Spektrums, bei der Maus etwa unterhalb von $6 \mathrm{kHz}$ und oberhalb von $48 \mathrm{kHz}$, finden sich nur etwa 7 bis 10 Synapsen pro innerer Haarzelle, während im Bereich des empfindlichsten Hörens zwischen 12 und $24 \mathrm{kHz}$ die Dichte mit 15 bis 18 Synapsen etwa doppelt so hoch ist. Eine ähnliche Situation findet sich in der Cochlea der mongolischen Wüstenrennmaus.

Offenbar ist es für die Funktion der Cochlea günstig, die mögliche Kapazität an Nervenfasern nicht gleichmäßig über das gesamte Spektrum zu verteilen, sondern gerade 
jenen Frequenzbereichen mehr Fasern zuzuordnen, die aufgrund der Übertragungseigenschaften von äußerem Ohr und Mittelohr und der aktiven Mikromechanik der Cochlea über eine höhere Empfindlichkeit verfügen. Mit Hilfe der zusätzlichen Fasern wäre es so möglich, den größeren Dynamikbereich hier abzubilden. Man kann spekulieren, ob sich der Bereich des besten Hörens - wie beispielsweise auch der Hauptsprachbereich des Menschen - evolutionär auf die Frequenzen der Signale mit der größten Relevanz für das Verhalten hin optimiert wurde.

\subsection{Verteilung der Synapsen in der Haarzelle}

Die afferenten Synapsen der zylinderförmigen Haarzellen sind vorzugsweise an deren basalem Pol lokalisiert. Wir haben hierzu eine Karte erstellt, die in einer Reihe von rekonstruierten immungefärbten Haarzellen die Synapsendichte sowie deren Verteilung in Abhängigkeit von der Seite (modiolus-nah/pfeilerzell-nah) aufzeigt (Publikation I, Abb. 2). Danach sind nahezu alle Synapsen am basalen Pol der Zelle unterhalb des Nukleus zu finden. Darüber hinaus findet sich jedoch keine präferentielle Anordnung im Hinblick auf den Winkel: Synapsen inserieren gleichverteilt an allen Seiten des basalen Pols. Dies trifft dabei sowohl bei apikalen als auch bei Haarzellen im mittleren Cochleaabschnitt zu. Hinweise auf eine möglicherweise bestehende differentielle Verteilung entsprechend der Empfindlichkeit der entsprechenden Faser, wie sie beispielsweise in der Katze bestehen (Merchan-Perez und Liberman, 1996), haben wir nicht gefunden. Dies ist konsistent mit dem Befund der konfokalen Calciummessungen (s. Abschnitt 3.5), die keine Ortspräferenz für Calciumdomänen unterschiedlicher Intensität in den Haarzellen aufdecken konnten.

\subsection{Struktur der afferenten Synapse}

Nach der Kartierung der Cochlea im Hinblick auf die Innervationsdichte und subzelluläre Synapsenlokalisation stellte sich die Frage, ob neben der Anzahl auch die Struktur der Synapsen entlang der Cochlea variiert. Die Größe einer einzelnen Synapse beträgt deutlich weniger als 1 Mikrometer, so daß zunächst geklärt werden mußte, welche Methoden überhaupt zur Untersuchung der einzelnen Elemente geeignet waren.

Nach wie vor das größte Auflösungsvermögen liefert die klassische TransmissionsElektronenmikroskopie. Wir untersuchten hiermit Synapsen in inneren Haarzellen der Maus am apikalen Ende und in der Mitte der Cochlea. An beiden Stellen findet sich der charakteristische elektronendichte Ribbon der Bändersynapse in vergleichbarer Größe. Die Anzahl und Größe der daran angehefteten synaptischen Vesikel scheint, wenn überhaupt, nur in geringem Maße unterschiedlich zu sein.

\subsection{Höchstauflösende optische Mikroskopie}

Wir haben nun drei für die Funktion der Synapse entscheidende Strukturen gewählt und diese mit spezifischen Antikörpern markiert: Auf der präsynaptischen Seite ist dies zunächst einmal der synaptische Ribbon. Einen Baustein hiervon bildet wie bereits erwähnt das Protein RIBEYE. Die Markierung mittels Immunfärbung zeigt dabei 
nicht nur die Lokalisation der Synapsen in der inneren Haarzelle sondern erlaubt auch eine Bestimmung der Größe - ein ausreichendes Auflösungsvermögen des Mikroskops vorausgesetzt. Wir haben daher die immungefärbten präsynaptischen Ribbons sowohl mit der 4Pi- als auch mit der STED-Mikroskopie an mehreren tonotopen Stellen untersucht. Danach variiert die Größe dieser präsynaptischen Struktur kaum: Einheitlich etwa 360 - $380 \mathrm{~nm}$ messen die Ribbons in ihrer längsten Ausdehnung.

Als weitere funktionell wichtige präsynaptische Struktur betrachteten wir die präsynaptischen Calciumkanäle, die durch ihr Öffnen nach Depolarisation die Transmitterausschüttung initiieren. Calciumkanäle sind an den Synapsen in sogenannten Clustern von geschätzt etwa 80 Kanälen (Brandt et al., 2005) konzentriert, wodurch ein räumlich definiertes Calciumsignal für den Freisetzungsapparat erzeugt wird. Die Größe der immunmarkierten Cluster bewegt sich an der Grenze der Auflösung konventioneller Lichtmikroskope, so daß wir auch hier die STED-Technik verwenden mußten: Die Form und Größe der Calciumkanalcluster können am besten durch ein flaches Ellipsoid mit einer langen Achse von $420 \pm 130 \mathrm{~nm}$ approximiert werden. Unterschiede in ihrer Größe an verschiedenen tonotopen Orten bestehen auch hier offenbar nicht.

Die dritte funktionell entscheidende Struktur, die wir vermessen haben ist das postsynaptische Cluster von Glutamatrezeptorkanälen, die das Transmittersignal wieder in elektrische Signale umwandeln. Die Dichte und Verteilung von Rezeptoren auf der postsynaptischen Seite hat direkten Einfluß auf die synaptische Stärke (Turrigiano, 2008) und kann daher möglicherweise den Schlüssel zur Erklärung der Signalübertragungseigenschaften der afferenten Nervenfasern liefern. Wir markierten Glutamatrezeptorkanäle des Subtyps GluR2/3 und bildeten diese mittels der STED-Technik in 2und 3-dimensionaler Auflösung ab. Die so gewonnenen Bilder der postsynaptischen Seite sind gleichermaßen wissenschaftlich wie ästhetisch faszinierend: Glutamatrezeptoren sind ringförmig mit einem Durchmesser von etwa $800 \mathrm{~nm}$ um den präsynaptischen Ribbon gruppiert und liegen so exakt gegenüber den vermuteten Stellen der Transmitterfreisetzung. Damit sind unsere Daten konsistent mit früheren Messungen (Matsubara et al., 1996), die mittels Elektronenmikroskopie an immunogoldgefärbten Synapsen ebenfalls Hinweise auf eine ringförmige Struktur des Glutamatrezeptorclusters ähnlicher Größe fanden.

\section{Funktion der afferenten Synapse}

Lieferte die Aufklärung der Morphologie der afferenten Synapse bereits eine ganze Reihe neuer Erkenntnisse über den Mechanismus des Hörens, so wollten wir diese Schlüsselstelle auch in Funktion untersuchen. Das Corti'sche Organ der Maus läßt sich unter Perfusion mit künstlicher Perilymphe für mehrere Stunden in vitro am Leben erhalten und mit funktionellen Methoden untersuchen. Dies haben wir zunächst mit der Patch-clamp-Technik getan. Sie ermöglicht unter anderem die Messung der präsynaptischen $\mathrm{Ca}^{2+}$-Ströme nach Depolarisation der Membran. Außerdem ist die Quantifizierung der so ausgelösten Transmitterfreisetzung über eine Messung der 
Membrankapazität möglich. Beide Parameter haben wir in inneren Haarzellen nahe dem Apex sowie am Übergang von der ersten zur zweiten Windung der Mauscochlea gemessen.

Innere Haarzellen in der Nähe des Apex zeigen im Mittel etwa 40\% weniger Transmitterfreisetzung als solche in den mittleren Abschnitten der Cochlea. Dabei entspricht die Differenz genau dem Faktor, der aufgrund der geringeren Synapsenzahl (apikal etwa 10, mitt-cochleär 14 Synapsen im Durchschnitt) zu erwarten ist. Wir gehen daher davon aus, daß im Mittel pro Synapse eine einheitliche Menge an Transmitter freigesetzt wird. Interessanterweise folgt der Calciumeinstrom nicht ebenso streng dieser Skalierung, was als ein Hinweis auf extrasynaptische Calciumkanäle gewertet werden könnte.

Über die Patch-Pipette läßt sich der Intrazellularraum mit Fluoreszenzfarbstoff füllen. Wir habe hierzu den Calciumindikator Fluo-5N gewählt, um mit Hilfe eines konfokalen Laserscanmikroskops den Calciumeinstrom an individuellen Synapsen nahe der präsynaptischen Membran abzubilden. Als Ergebnis konnten wir feststellen, daß nach den vorangegangenen Untersuchungen wenig überraschend - die Unterschiede in der Dynamik und Ausdehnung des präsynaptischen Calciumsignals sich an verschiedenen tonotopen Stellen der Cochlea im Mittel wenig unterscheiden. Überraschenderweise jedoch zeigte sich der Calciumeinstrom an verschiedenen Synapsen innerhalb der selben inneren Haarzelle von erheblicher Variabilität. Wir vermuten, daß mit dem Calciumsignal auch die Transmitterfreisetzung von Synapse zu Synapse deutliche Unterschiede aufweist. Dies könnte einen Mechanismus darstellen, der das breite Spektrum an unterschiedlichen Empfindlichkeiten und Aktionspotentialfrequenzen in den afferenten Nervenfasern bedingt und so zur Codierung von Schallsignalen in einem breiten Dynamikbereich beiträgt.

\subsection{Calcium-binding Proteins und calciumabhängige Calciumstrominaktivierung}

Die in der zweiten Publikation vorgestellten Arbeiten zur Funktion der calciumbinding proteins (Cui et al., 2007) wurden in Zusammenarbeit mit dem Labor von Amy Lee, Emory University, Atlanta, durchgeführt. Ausgangspunkt war die Frage, ob die ungewöhnlich langsame CDI der Ca $a_{v}$ 1.3-Calciumkanäle der inneren Haarzellen durch ein oder mehrere Vertreter der CaBP-Familie vermittelt wird.

Schwerpunktmäßig wurde in dieser Arbeit die Funktion von CaBP4 untersucht. Von den Kollegen konnte gezeigt werden, daß CaBP4 funktionell mit dem Cav1.3-Kanal interagiert. Des weiteren wurde eine mutante Mauslinie mit einem knock-out für das CaBP4 generiert. Wir konnten nun mit immunhistochemischen Methoden zeigen, daß CaBP4 wie auch CaBP1, 2 und 5 im Corti'schen Organ bei Einsetzen des Hörens exprimiert wird (Abbildung 1 und 5).

Untersuchungen von akustisch evozierten Potentialen sowie otoakustischen Emissionen zeigten, daß die Funktion von Innenohr und Hörbahn in CaBP4-Mutanten keine 
Defizite gegenüber Wildtyp-Tieren aufwiesen (Abbildung 4). Zur Bestimmung der Funktion von präsynaptischen Calciumkanälen und der Freisetzung von Transmitter führten wir Patch-clamp-Messungen an inneren Haarzellen durch. Sowohl Calciumstrom als auch Cav1.3-vermittelter Strom von Barium zeigten dabei in Größe und Kinetik keine signifikanten Unterschiede zwischen Mutante und Wildtyp. Dies trifft auch auf die Freisetzung von Transmitter zu, die in der Mutante unverändert scheint und so das normale Hörvermögen erklärt (Abbildung 4).

Spielt CaBP4 nun überhaupt eine Rolle bei der Modulation der Calciumströme innerer Haarzellen? Messungen von Calciumströmen in rekombinanten Systemen (Abbildung 3 und 6) legen nun den Schluß nahe, daß die Interaktion von Cav1.3-Kanälen mit CaBP4, ähnlich wie auch CaBP2 und CaBP5, funktionell nur geringe Auswirkungen besitzt. Anders scheint dies jedoch für $\mathrm{CaBP} 1$ zu sein: Hier weisen Calciumströme bei Ko-Expression eine deutlich verminderte CDI auf.

Wir haben daher das Expressionsmuster von CaBP1 in inneren Haarzellen untersucht. Interessanterweise finden sich CaBP1-immunpositive Punkte kolokalisiert mit dem präsynaptischen Marker RIBEYE/CtBP2 (Abbildung 5), so daß wir dieses Mitglied der CaBP-Familie für einen guten Kandidaten des Modulators der Cav1.3-Kinetik in inneren Haarzellen halten. 
Wir konnten zeigen, daß die afferenten Fasern des Hörnerven sich nicht gleichmäßig auf die gesamte Cochlea verteilen, sondern die tonotopen Bereiche mit der höchsten Empfindlichkeit - etwa in deren Mitte - zirka zwei- bis dreimal mehr Synapsen und damit Fasern pro innerer Haarzelle besitzen als jene an den Enden. Damit könnte beispielsweise dem größeren hier zu codierenden Dynamikumfang Rechnung getragen werden.

Die Verteilung der Synapsen innerhalb der inneren Haarzellen konzentriert sich an deren basalem Pol. Eine bevorzugte Lokalisation an neuraler oder abneuraler Seite konnte nicht festgestellt werden. Dies wird auch durch die Ergebnisse der konfokalen intrazellulären Calciummessungen unterstützt. Aufbau und Funktion der einzelnen Synapse zeigen nur geringe tonotope Unterschiede, die Synapsen erscheinen in ihrem grundlegenden Aufbau einem „Einheitsentwurf“ zu folgen. Auch die Menge an freigesetztem Neurotransmitter pro Synapse erscheint im Mittel vergleichbar. Ob die mit unseren Methoden detektierbaren geringen Unterschiede auch funktionell relevant sind, müssen weitere Experimente, möglicherweise mit gleichzeitiger prä- und postsynaptischer Ableitung, zeigen.

Präsynaptische Cav1.3-Calciumkanäle in inneren Haarzellen zeigen eine ungewöhnlich geringe calciumabhängige Calciumstrominaktivierung, die wahrscheinlich von einem Mitglied der calcium-binding-protein-Familie, nämlich CaBP1, vermittelt wird. Das Fehlen von CaBP4 dagegen scheint nur minimalen Einfluß auf die Funktion der inneren Haarzellen und der gesamten Hörbahn zu haben. 


\section{$5 \quad$ Literatur}

Abbe E (1873): Beiträge zur Theorie des Mikroskops und der mikroskopischen Wahrnehmung. Arch Mikroskop Anat 9:413-468.

Bekesy Gv: Experiments in Hearing: McGraw-Hill, New York 1960

Beutner D, Voets T, Neher E, Moser T (2001): Calcium dependence of exocytosis and endocytosis at the cochlear inner hair cell afferent synapse. Neuron 29:681-690.

Brandt A, Striessnig J, Moser T (2003): CaV1.3 channels are essential for development and presynaptic activity of cochlear inner hair cells. J Neurosci 23:10832-10840.

Brandt A, Khimich D, Moser T (2005): Few CaV1.3 channels regulate the exocytosis of a synaptic vesicle at the hair cell ribbon synapse. J Neurosci 25:11577-11585.

Catterall WA, Few AP (2008): Calcium channel regulation and presynaptic plasticity. Neuron 59:882-901.

Cheatham MA, Low-Zeddies S, Naik K, Edge R, Zheng J, Anderson CT, Dallos P (2009): A chimera analysis of prestin knock-out mice. J Neurosci 29:12000-12008.

Cui G, Meyer AC, Calin-Jageman I, Neef J, Haeseleer F, Moser T, Lee A (2007): Ca2+-binding proteins tune Ca2+-feedback to Cav1.3 channels in mouse auditory hair cells. J Physiol (Lond) 585:791-803.

Dallos P, Harris D (1978): Properties of auditory nerve responses in absence of outer hair cells. J Neurophysiol 41:365-383.

Drexl M, Lagarde MMM, Zuo J, Lukashkin AN, Russell IJ (2008): The role of prestin in the generation of electrically evoked otoacoustic emissions in mice. J Neurophysiol 99:16071615.

Evans EF (1972): The frequency response and other properties of single fibres in the guinea-pig cochlear nerve. J Physiol (Lond) 226:263-287.

Eybalin M, Caicedo A, Renard N, Ruel J, Puel J-L (2004): Transient Ca2+-permeable AMPA receptors in postnatal rat primary auditory neurons. Eur J Neurosci 20:2981-2989.

Fettiplace R (2009): Defining features of the hair cell mechanoelectrical transducer channel. Pflugers Arch 458:1115-1123.

Francis HW, Rivas A, Lehar M, Ryugo DK (2004): Two types of afferent terminals innervate cochlear inner hair cells in C57BL/6J mice. Brain Res 1016:182-194. 
Frank T, Khimich D, Neef A, Moser T (2009): Mechanisms contributing to synaptic Ca2+ signals and their heterogeneity in hair cells. Proc Natl Acad Sci USA 106:4483-4488.

Goutman JD, Glowatzki E (2007): Time course and calcium dependence of transmitter release at a single ribbon synapse. Proc Natl Acad Sci USA 104:16341-16346.

Haeseleer F, Sokal I, Verlinde CL, Erdjument-Bromage H, Tempst P, Pronin AN, Benovic JL, Fariss RN, Palczewski K (2000): Five members of a novel Ca(2+)-binding protein (CABP) subfamily with similarity to calmodulin. J Biol Chem 275:1247-1260.

Hamill OP, Marty A, Neher E, Sakmann B, Sigworth FJ (1981): Improved patch-clamp techniques for high-resolution current recording from cells and cell-free membrane patches. Pflugers Arch 391:85-100.

Hell SW (2007): Far-field optical nanoscopy. Science $\underline{316}$ :1153-1158.

Hell SW, Wichmann J (1994): Breaking the diffraction resolution limit by stimulated-emission stimulated-emission-depletion fluorescence microscopy. Opt Lett 19:780-782.

Hell SW, Schmidt R, Egner A (2009): Diffraction-unlimited three-dimensional optical nanoscopy with opposing lenses. Nat Photonics $\underline{3}: 381-387$.

Henry KR, Lepkowski CM (1978): Evoked potential correlates of genetic progressive hearing loss. Age-related changes from the ear to the inferior colliculus of C57BL/6 and CBA/J mice. Acta Otolaryngol 86:366-374.

Howard J, Roberts WM, Hudspeth AJ (1988): Mechanoelectrical transduction by hair cells. Ann Rev Biophys Chem 17:99-124.

Hudspeth AJ (1989): How the ear's works work. Nature 341:397-404.

Khimich D, Nouvian R, Pujol R, tom Dieck S, Egner A, Gundelfinger ED, Moser T (2005): Hair cell synaptic ribbons are essential for synchronous auditory signalling. Nature $\underline{434}: 889$ 894.

Kiang N-S, Watanabe T, Thomas E, Clarke L: Discharge Patterns of Single Fibers in the Cat's Auditory Nerve: MIT Press, Cambridge, MA 1965

Kiang NY, Liberman MC, Levine RA (1976): Auditory-nerve activity in cats exposed to ototoxic drugs and high-intensity sounds. Ann Otol Rhinol Laryngol 85:752-768.

Kiang NY, Rho JM, Northrop CC, Liberman MC, Ryugo DK (1982): Hair-cell innervation by spiral ganglion cells in adult cats. Science 217:175-177. 
Koschak A, Reimer D, Huber I, Grabner M, Glossmann H, Engel J, Striessnig J (2001): alpha 1D (Cav1.3) subunits can form l-type Ca2+ channels activating at negative voltages. J Biol Chem 276:22100-22106.

Kros CJ, Ruppersberg JP, Rüsch A (1998): Expression of a potassium current in inner hair cells during development of hearing in mice. Nature 394:281-284.

Lee A, Westenbroek RE, Haeseleer F, Palczewski K, Scheuer T, Catterall WA (2002): Differential modulation of $\mathrm{Ca}(\mathrm{v}) 2.1$ channels by calmodulin and $\mathrm{Ca} 2+$-binding protein 1 . Nat Neurosci 5:210-217.

Li G, Keen E, Andor-Ardo D, Hudspeth A, von Gersdorff H (2009): The unitary event underlying multiquantal EPSCs at a hair cell's ribbon synapse. J Neurosci 29:7558-7568.

Liberman MC (1978): Auditory-nerve response from cats raised in a low-noise chamber. J Acoust Soc Am 63:442-455.

Liberman MC (1980): Morphological differences among radial afferent fibers in the cat cochlea: an electron-microscopic study of serial sections. Hear Res $\underline{3}: 45-63$.

Liberman MC (1982a): Single-neuron labeling in the cat auditory nerve. Science 216:1239-1241.

Liberman MC (1982b): The cochlear frequency map for the cat: labeling auditory-nerve fibers of known characteristic frequency. J Acoust Soc Am 72:1441-1449.

Liberman MC, Dodds LW, Pierce S (1990): Afferent and efferent innervation of the cat cochlea: quantitative analysis with light and electron microscopy. J Comp Neurol 301:443-460.

Lindau M, Neher E (1988): Patch-clamp techniques for time-resolved capacitance measurements in single cells. Pflugers Arch 411:137-146.

Matsubara A, Laake JH, Davanger S, Usami S, Ottersen OP (1996): Organization of AMPA receptor subunits at a glutamate synapse: a quantitative immunogold analysis of hair cell synapses in the rat organ of Corti. J Neurosci 16:4457-4467.

Merchan-Perez A, Liberman MC (1996): Ultrastructural differences among afferent synapses on cochlear hair cells: correlations with spontaneous discharge rate. J Comp Neurol 371:208-221.

Meyer AC, Frank T, Khimich D, Hoch G, Riedel D, Chapochnikov NM, Yarin YM, Harke B, Hell SW, Egner A, Moser T (2009): Tuning of synapse number, structure and function in the cochlea. Nat Neurosci 12:444-453.

Michna M, Knirsch M, Hoda J-C, Muenkner S, Langer P, Platzer J, Striessnig J, Engel J (2003): Cav1.3 (alpha1D) Ca2+ currents in neonatal outer hair cells of mice. J Physiol (Lond) 553:747-758. 
Møller A: Hearing. Anatomy, Physiology, and Disorders of the Auditory System, 2nd Edition: Academic Press, Burlington, MA 2006

Moore DR, Hine JE (1992): Rapid development of the auditory brainstem response threshold in individual ferrets. Brain Res Dev Brain Res 66:229-235.

Moser T, Beutner D (2000): Kinetics of exocytosis and endocytosis at the cochlear inner hair cell afferent synapse of the mouse. Proc Natl Acad Sci USA 97:883-888.

Moser T, Brandt A, Lysakowski A (2006): Hair cell ribbon synapses. Cell Tissue Res $\underline{326}$ :347359.

Mullen L, Ryan A: Transgenic mice: genome manipulation and induced mutations. In: Handbook of Mouse Auditory Research From Behavior to Molecular Biology (Willott J, ed), pp 457- 474. Boca Raton, FL: CRC Press, Boca Raton, FL 2001

Müller M (1996): The cochlear place-frequency map of the adult and developing Mongolian gerbil. Hear Res 94:148-156.

Müller M, von Hünerbein K, Hoidis S, Smolders JWT (2005): A physiological place-frequency map of the cochlea in the CBA/J mouse. Hear Res 202:63-73.

Neef J, Gehrt A, Bulankina AV, Meyer AC, Riedel D, Gregg RG, Strenzke N, Moser T (2009): The $\mathrm{Ca} 2+$ channel subunit beta2 regulates $\mathrm{Ca} 2+$ channel abundance and function in inner hair cells and is required for hearing. J Neurosci 29:10730-10740.

Ngan EM, May BJ (2001): Relationship between the auditory brainstem response and auditory nerve thresholds in cats with hearing loss. Hear Res 156:44-52.

Ohlemiller KK, Echteler SM (1990): Functional correlates of characteristic frequency in single cochlear nerve fibers of the Mongolian gerbil. J Comp Physiol A 167:329-338.

Pawley J: Handbook of Biological Confocal Microscopy, 3 Edition: Springer, Berlin 2006

Peterson BZ, DeMaria CD, Adelman JP, Yue DT (1999): Calmodulin is the Ca2+ sensor for $\mathrm{Ca} 2+$-dependent inactivation of L-type calcium channels. Neuron 22:549-558.

Platzer J, Engel J, Schrott-Fischer A, Stephan K, Bova S, Chen H, Zheng H, Striessnig J (2000): Congenital deafness and sinoatrial node dysfunction in mice lacking class D L-type Ca2+ channels. Cell 102:89-97.

Qin N, Olcese R, Bransby M, Lin T, Birnbaumer L (1999): Ca2+-induced inhibition of the cardiac Ca2+ channel depends on calmodulin. Proc Natl Acad Sci USA $\underline{96: 2435-2438 .}$

Roux I, Safieddine S, Nouvian R, Grati Mh, Simmler M-C, Bahloul A, Perfettini I, Le Gall M, Rostaing P, Hamard G, Triller A, Avan P, Moser T, Petit C (2006): Otoferlin, defective 
in a human deafness form, is essential for exocytosis at the auditory ribbon synapse. Cell 127:277-289.

Ruel J, Bobbin RP, Vidal D, Pujol R, Puel JL (2000): The selective AMPA receptor antagonist GYKI 53784 blocks action potential generation and excitotoxicity in the guinea pig cochlea. Neuropharmacology 39:1959-1973.

Ryan A, Dallos P (1975): Effect of absence of cochlear outer hair cells on behavioural auditory threshold. Nature 253:44-46.

Sakmann B, Neher E: Single-channel recording: Springer, Berlin 1995

Schmitz F, Konigstorfer A, Sudhof T (2000): RIBEYE, a component of synaptic ribbons: a protein's journey through evolution provides insight into synaptic ribbon function. Neuron 28:857-872.

Schnee ME, Ricci AJ (2003): Biophysical and pharmacological characterization of voltage-gated calcium currents in turtle auditory hair cells. J Physiol (Lond) 549:697-717.

Schneggenburger R, Neher E (2000): Intracellular calcium dependence of transmitter release rates at a fast central synapse. Nature $\underline{406: 889-893}$.

Song H, Nie L, Rodriguez-Contreras A, Sheng Z-H, Yamoah EN (2003): Functional interaction of auxiliary subunits and synaptic proteins with $\mathrm{Ca}(\mathrm{v}) 1.3$ may impart hair cell $\mathrm{Ca} 2+$

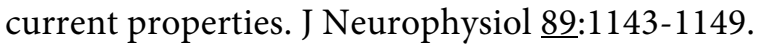

Spoendlin H (1969): Innervation patterns in the organ of corti of the cat. Acta Otolaryngol 67:239-254.

Spoendlin H (1985): Anatomy of cochlear innervation. Am J Otolaryngol ㅁ:453-467.

Stamataki S, Francis HW, Lehar M, May BJ, Ryugo DK (2006): Synaptic alterations at inner hair cells precede spiral ganglion cell loss in aging C57BL/6J mice. Hear Res 221:104-118.

Sterling P, Matthews G (2005): Structure and function of ribbon synapses. Trends Neurosci 28:20-29.

Taberner AM, Liberman MC (2005): Response properties of single auditory nerve fibers in the mouse. J Neurophysiol 93:557-569.

Tasaki I (1954): Nerve impulses in individual auditory nerve fibers of guinea pig. J Neurophysiol 17:97-122.

Tucker T, Fettiplace R (1995): Confocal imaging of calcium microdomains and calcium extrusion in turtle hair cells. Neuron 15:1323-1335. 
Turrigiano GG (2008): The self-tuning neuron: synaptic scaling of excitatory synapses. Cell 135:422-435.

Uzuka Y, Furuta T, Yamaoka M, Ohnishi T, Tsubone H, Sugano S (1996): Threshold changes in auditory brainstem response (ABR) due to the administration of kanamycin in dogs. Exp Anim $\underline{45}: 325-331$.

Willott JF (1986): Effects of aging, hearing loss, and anatomical location on thresholds of inferior colliculus neurons in C57BL/6 and CBA mice. J Neurophysiol 56:391-408.

Wolski LF, Anderson RC, Bowles AE, Yochem PK (2003): Measuring hearing in the harbor seal (Phoca vitulina): comparison of behavioral and auditory brainstem response techniques. J Acoust Soc Am 113:629-637.

Xu W, Lipscombe D (2001): Neuronal Ca(V)1.3alpha(1) L-type channels activate at relatively hyperpolarized membrane potentials and are incompletely inhibited by dihydropyridines. J Neurosci 21:5944-5951.

Yang PS, Alseikhan BA, Hiel H, Grant L, Mori MX, Yang W, Fuchs PA, Yue DT (2006): Switching of $\mathrm{Ca} 2+$-dependent inactivation of $\mathrm{Ca}(\mathrm{v}) 1.3$ channels by calcium binding proteins of auditory hair cells. J Neurosci 26:10677-10689.

Zheng QY, Johnson KR, Erway LC (1999): Assessment of hearing in 80 inbred strains of mice by ABR threshold analyses. Hear Res 130:94-107.

Zhou H, Kim S-A, Kirk EA, Tippens AL, Sun H, Haeseleer F, Lee A (2004): Ca2+-binding protein-1 facilitates and forms a postsynaptic complex with Cav1.2 (L-type) Ca2+ channels. J Neurosci 24:4698-4708. 


\section{Publikationen}

\section{Publikation I:}

Meyer AC, Frank T, Khimich D, Hoch G, Riedel D, Chapochnikov NM, Yarin YM, Harke B, Hell SW, Egner A, Moser T (2009):

Tuning of synapse number, structure and function in the cochlea.

Nature Neuroscience 12:444-453

DOI: $10.1038 / \mathrm{nn} .2293$

PMID: 19270686

\section{Publikation II:}

Cui G, Meyer AC, Calin-Jageman I, Neef J, Haeseleer F, Moser T, Lee A (2007):

$C a^{2+}$-binding proteins tune $\mathrm{Ca}^{2+}$-feedback to $\mathrm{Ca}_{V} 1.3$ channels in mouse auditory hair cells.

Journal of Physiology 585:791-803

DOI: 10.1113/jphysiol.2007.142307

PMID: 17947313 


\section{Danksagung}

An erster Stelle möchte ich mich bei Prof. Tobias Moser für die Anregung, Unterstützung, Begleitung und persönliche Zusammenarbeit im Rahmen dieser Arbeit bedanken.

Für die besondere Unterstützung und Hilfe in Mikroskopie-Fragen möchte ich Alexander Egner ganz herzlich danken. Ebenfalls danke ich Thomas Frank, Darina Khimich, Gerhard Hoch, Yury Yarin, Nikolai Chapochnikov, Christian Rüdiger, Dietmar Riedel und Benjamin Harke für die produktive und nette Zusammenarbeit. Der Arbeitsgruppe vom Max-Planck-Institut am Faßberg um Prof. Stefan Hell danke ich außerdem für die Unterstützung bei dieser Arbeit.

Die Zusammenarbeit mit der Arbeitsgruppe um Amy Lee an der Emory University war stets kollegial und freundlich; an dieser Stelle dafür herzlichen Dank.

Dem Direktor der Abteilung Hals-Nasen-Ohrenheilkunde Prof. Christoph Matthias möchte ich an dieser Stelle für die Förderung meiner Forschungstätigkeit danken. 


\section{Lebenslauf}

Ich wurde am 1. Februar 1975 als Sohn von Karin und Dipl.-Ing. Karl-Wilhelm Meyer in Osnabrück geboren. Meine Schulausbildung erhielt ich in Osnabrück, wo ich 1994 auch das Abitur am Graf-Stauffenberg-Gymnasium erwarb.

Zum Wintersemester 1994/95 schrieb ich mich im Diplomstudiengang Physik an der Universität Osnabrück ein. 1996 bestand ich das Vordiplom in Physik, Mathematik und Informatik. Zum Wintersemester 1996/97 wechselte ich in den gleichen Studiengang der Georg-August-Universität Göttingen, an der ich 1999 die Diplom-Prüfung in Physik mit „sehr gut“ bestand. Die Diplomarbeit hatte ich zuvor von September 1997 bis Mai 1999 am Max-Planck-Institut für biophysikalische Chemie unter Anleitung von Prof. Dr. E. Neher zum Thema „Untersuchungen zur Freisetzungswahrscheinlichkeit an einer zentralen Synapse" angefertigt.

In der gleichen Arbeitsgruppe schloß sich von 2000 bis 2003 eine Promotionsarbeit in Biophysik mit dem Thema „Untersuchungen zur Funktion der präsynaptischen Zytomatrixproteine Piccolo und Bassoon“ an, mit der ich 2004 mit „magna cum laude“ zum Dr. rer. nat. promoviert wurde.

Von 1994 bis 1999 war ich Stipendiat der Studienstiftung des Deutschen Volkes. Während meiner Promotion 2000 bis 2003 erhielt ich ein Stipendium des Boehringer Ingelheim Fonds.

Zum Sommersemester 1998 schrieb ich mich zusätzlich in den Studiengang Humanmedizin an der Georg-August-Universität Göttingen ein. Das Studium der Medizin schloß ich 2005 nach dem praktischen Jahr mit dem dritten Staatsexamen (Gesamtnote „sehr gut") ab und erhielt die Approbation.

2005 war ich als Post-doc am Baylor College of Medicine, Houston, Texas, in der Arbeitsgruppe von Dr. Ch. Rosenmund tätig. Seit 2005 arbeite ich als wissenschaftlicher Mitarbeiter und Arzt in Weiterbildung an der Hals-Nasen-Ohrenklinik der Universitätsmedizin Göttingen.

Von der Arbeitsgemeinschaft deutschsprachiger Audiologen und Neurootologen (ADANO) der Deutschen Gesellschaft für Hals-Nasen-Ohrenheilkunde wurde mir der Innovationspreis 2009 verliehen.

Ich bin seit 2008 verheiratet mit der Zahnärztin Sonja Meyer-Rollmann. Unsere Tochter Elisa Berenike wurde am 15. September 2009 geboren. 


\section{Publikationen}

Schneggenburger R, Meyer AC, Neher E (1999): Released fraction and total size of a pool of immediately available transmitter quanta at a calyx synapse. Neuron 23:399-409.

Meyer AC, Neher E, Schneggenburger R (2001): Estimation of quantal size and number of functional active zones at the calyx of held synapse by nonstationary EPSC variance analysis. J Neurosci 21:7889-7900.

Fernández-Chacón R, Shin O-H, Königstorfer A, Matos MF, Meyer AC, Garcia J, Gerber SH, Rizo J, Südhof TC, Rosenmund C (2002): Structure/function analysis of Ca2+ binding to the C2A domain of synaptotagmin 1. J Neurosci 22:8438-8446.

Altrock WD*, tom Dieck $\mathrm{S}^{\star}$, Sokolov $\mathrm{M}^{\star}$, Meyer AC*, Sigler A, Brakebusch C, Fässler R, Richter K, Boeckers TM, Potschka H, Brandt C, Löscher W, Grimberg D, Dresbach T, Hempelmann A, Hassan H, Balschun D, Frey JU, Brandstätter JH, Garner CC, Rosenmund C, Gundelfinger ED (2003): Functional inactivation of a fraction of excitatory synapses in mice deficient for the active zone protein bassoon. Neuron $\underline{37: 787-800 .}$

Moser T, Strenzke N, Meyer A, Lesinski-Schiedat A, Lenarz T, Beutner D, Foerst A, Lang-Roth R, von Wedel H, Walger M, Gross M, Keilmann A, Limberger A, Steffens T, Strutz J (2006): Diagnose und Therapie der auditorischen Synaptopathie. HNO 544:833-839.

Pang ZP*, Shin O-H* $\mathrm{H}^{\star}$, Meyer AC*, Rosenmund C, Südhof TC (2006): A gain-of-function mutation in synaptotagmin-1 reveals a critical role of $\mathrm{Ca} 2+$-dependent soluble $\mathrm{N}$-ethylmaleimidesensitive factor attachment protein receptor complex binding in synaptic exocytosis. J Neurosci 26:12556-12565.

Cui G*, Meyer AC*, Calin-Jageman I, Neef J, Haeseleer F, Moser T, Lee A (2007): Ca2+binding proteins tune $\mathrm{Ca} 2+$-feedback to Cav1.3 channels in mouse auditory hair cells. J Physiol (Lond) 585:791-803.

Gerber SH, Rah J-C, Min S-W, Liu X, de Wit H, Dulubova I, Meyer AC, Rizo J, Arancillo M, Hammer RE, Verhage M, Rosenmund C, Südhof TC (2008): Conformational switch of syntaxin-1 controls synaptic vesicle fusion. Science $\underline{321: 1507-1510 .}$

Strenzke N, Pauli-Magnus D, Meyer A, Brandt A, Maier H, Moser T (2008): Update zur Physiologie und Pathophysiologie des Innenohres. HNO 흐:27-36.

Meyer AC*, Frank T*, Khimich D*, Hoch G, Riedel D, Chapochnikov NM, Yarin YM, Harke B, Hell SW, Egner A, Moser T (2009): Tuning of synapse number, structure and function in the cochlea. Nat Neurosci 12:444-453.

Neef J, Gehrt A, Bulankina AV, Meyer AC, Riedel D, Gregg RG, Strenzke N, Moser T (2009): The $\mathrm{Ca} 2+$ channel subunit beta2 regulates $\mathrm{Ca} 2+$ channel abundance and function in inner hair cells and is required for hearing. J Neurosci 29:10730-10740. 